\title{
Unsuccessful Progression of Sacral Neuromodulation From the Evaluation to the Implantation Stage: A Single-Center Experience
}

\author{
Mohammad A. Alghafees ${ }^{1}$, Mohammed K. Alageel ${ }^{1}$, Meshari A. Alqahtani ${ }^{1}$, Yahia Alghazwani ${ }^{2}$ \\ 1. College of Medicine, King Saud Bin Abdulaziz University for Health Sciences, Riyadh, SAU 2. Urology, King Abdulaziz \\ Medical City, Riyadh, SAU
}

Corresponding author: Mohammad A. Alghafees, alghafees687@gmail.com

\begin{abstract}
Introduction

Over the years, sacral neuromodulation (SNM) has become an established and effective treatment for chronic urinary system retention and incontinence. The process of SNM is performed in two stages, the first is an evaluation phase and the second an implant phase. This study aimed to assess the rate of failure of progression from the evaluation to the implantation stage and the factors predicting the outcome of this commonplace procedure.
\end{abstract}

\section{Materials and methods}

This retrospective cross-sectional study took place at King Abdulaziz Medical City (KAMC), Riyadh, Saudi Arabia. All the patients who underwent SNM implantation from January 1, 2016 to January 1, 2021 were included. Patients younger than 14 years and patients who had the SNM implantation in a different hospital and were only followed-up at KAMC were excluded. Patient-related information were extracted from the BESTCare system. Frequency and percentage were used for the categorical variables, and the mean, median, and standard deviation to display the continuous variables. Chi-square test and Mann-Whitney U test were used to test for the association of the categorical variables.

\section{Results}

Among 28 patients, $46.4 \%(n=13)$ failed to progress from the evaluation phase to the implantation phase. Gender, age, having a co-morbidity, and SNM indication were not significant factors for predicting the outcome of the SNM evaluation phase.

\section{Conclusion}

The observed failure rate was marginally higher than the ones detected in other studies. Although no significant association was detected between evaluation stage failure and the assigned predictors, the results need to be interpreted with caution due to the small population size. Larger multicenter studies need to be done in order to investigate the link between patient characteristics and the efficacy of SNM.

Review began 07/15/2021 Review ended 08/05/2021 Published 08/05/2021

\section{() Copyright 2021}

Alghafees et al. This is an open access article distributed under the terms of the Creative Commons Attribution License CC-BY 4.0., which permits unrestricted use, distribution, and reproduction in any medium, provided the original author and source are credited.
Establishing a concrete evidence would further refine the targeted patient population and indications for SNM.

\author{
Categories: Urology \\ Keywords: urology, sacral neuromodulation, bladder dysfunction, neuro-urology, saudi arabia
}

\section{Introduction}

Over the years, sacral neuromodulation (SNM) has become an established and effective treatment for chronic urinary system retention and incontinence. The process of sacral neuromodulation is performed in two stages, the first is an evaluation phase and the second an implant phase. In the evaluation phase, the physician assesses whether or not the symptoms will be adequately treated with SNM. Also, it gives a chance for the patient to modify their lifestyle and daily life with the SNM device implanted. On average, the evaluation phase takes between two to eight weeks [1]. In some instances, the evaluation phase could fail due to many reasons, such as infection, breaking of lead, or patient dissatisfaction [2]. However, there is emerging evidence that other parameters, such as age, gender, SNM indication, and body mass index (BMI) could have a role in increasing the likelihood of failure to progress to the implantation stage.

In Saudi Arabia, with the increasing use of SNM, urologists are more likely to encounter evaluation stage failures, the presented study aimed to assess the rate of failure of progression from the evaluation to the implantation stage and its predictors among patients undergoing sacral neuromodulation in King Abdulaziz Medical City (KAMC), a tertiary academic hospital in Riyadh, Saudi Arabia. Further studies in the region 


\section{Cureus}

exploring the failure of SNM treatment among patients would further refine the targeted patient population and indications for SNM consequently improving patient outcomes.

\section{Materials And Methods}

This retrospective cross-sectional study was conducted at KAMC, Riyadh, Saudi Arabia. All the patients who underwent SNM implantation from January 1, 2016 to January 1, 2021 were included. Patients younger than 14 years and patients who had the SNM implantation in a different hospital and were only followed-up at KAMC were excluded.

Demographic information, comorbidities, the indication for SNM, and failure at the evaluation stage of implantation were extracted from the BESTCare system (ezCareTech, South Korea). The data were entered in Microsoft Excel 2019 (Microsoft Corporation, WA, USA) and the statistical analysis was done with the Statistical Package for the Social Sciences (SPSS) version 23.0 (IBM Corporation, Armonk, NY, USA). Frequency and percentage are used to display the categorical variables, and the mean, median, and standard deviation to display the continuous variables. Chi-square test and Mann-Whitney U-test were used to test for association of gender, age, co-morbidity presence, and SNM indication with the outcome of the evaluation stage in SNM. The level of significance was set at 0.05 .

The patients' confidentiality and anonymity were ensured, as serial numbers replaced the medical record numbers. The data was accessed and used by only the research team. The Institutional Review Board of King Abdullah International Medical Research Center, the Ministry of National Guard-Health Affairs, Riyadh, Kingdom of Saudi Arabia, approved the study with approval number NRC21R/095/03.

\section{Results}

The final population size was 28 patients. Table 1 displays the sociodemographic profile of the sample. Of the population, $46.4 \%(n=13)$ were males and $53.6 \%(n=15)$ were females. The minimum age was 17 years, the maximum was 73 years, and the mean age was $37.14+14.62$ years.

\begin{tabular}{|c|c|c|}
\hline Demographical characteristic & $\mathbf{n}$ & $\%$ \\
\hline \multicolumn{3}{|l|}{ Gender } \\
\hline Male & 13 & 46.4 \\
\hline Female & 15 & 53.6 \\
\hline \multicolumn{3}{|l|}{ Age } \\
\hline Mean & 37.14 & \\
\hline Standard deviation & 14.62 & \\
\hline Minimum & 17 & \\
\hline Maximum & 73 & \\
\hline
\end{tabular}

TABLE 1: Socio-demographic profile of the patients $(n=28)$.

Figure 1 displays the co-morbidities occurring in the sample. More than half $(\mathrm{n}=16,57.1 \%)$ were medically fit, $10.7 \%(\mathrm{n}=3)$ had type 1 diabetes, $10.7 \%(\mathrm{n}=3)$ chronic kidney disease, $7.1 \%(\mathrm{n}=2)$ had hypertension, $7.1 \%$ $(n=2)$ had dyslipidemia, 3.6\% $(n=1)$ congestive heart failure, $3.6 \%(n=1)$ generalized anxiety disorder, $3.6 \%$ $(n=1)$ had peripheral vascular disease, $3.6 \%(n=1)$ had type 2 diabetes, and $3.6 \%(n=1)$ had a cerebrovascular accident. 


\section{Cureus}

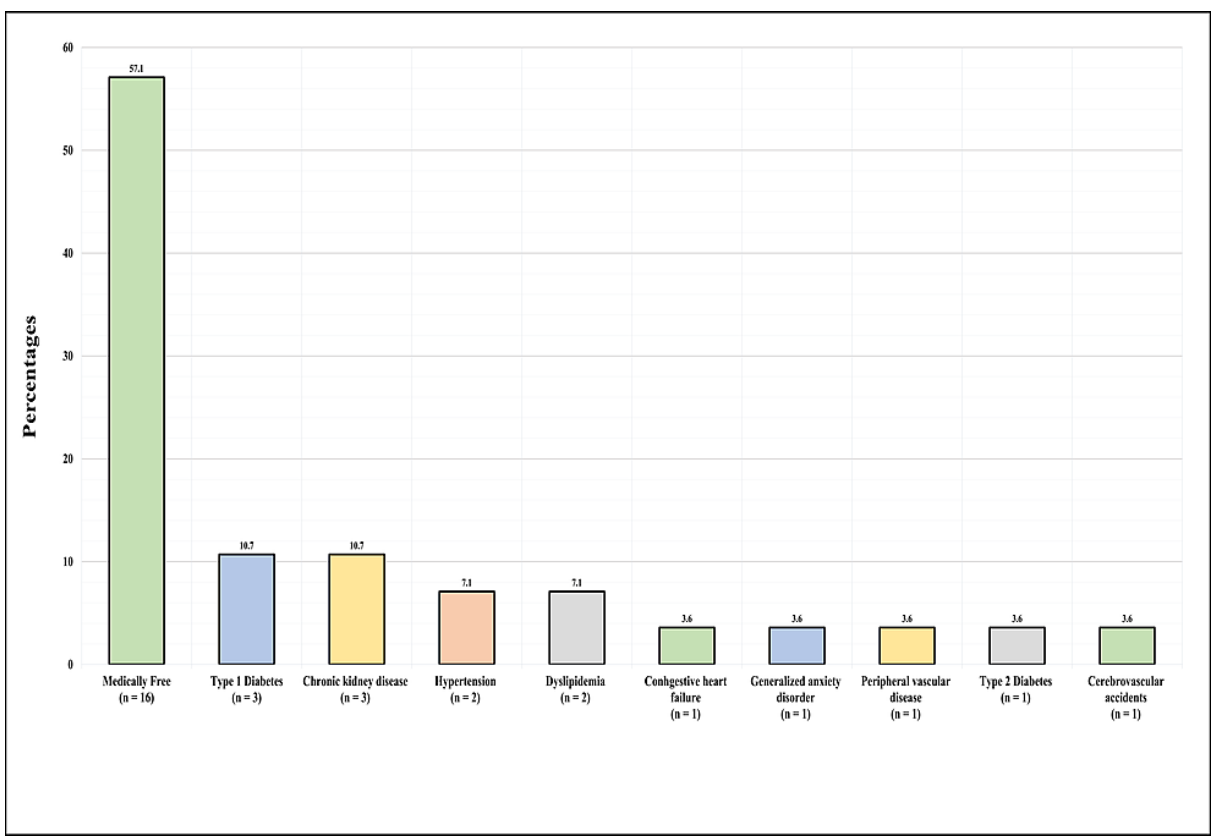

FIGURE 1: Presence of comorbidities among participants.

Figure 2 presents the patients' diagnoses. The highest proportion, $28.6 \%(\mathrm{n}=8)$, had an idiopathic bladder dysfunction, $21.4 \%(\mathrm{n}=6)$ had a neurogenic bladder due to a spinal cord injury, $14.3 \%(\mathrm{n}=4)$ had an overactive bladder, 7.1\% $(\mathrm{n}=2)$ had urinary incontinence, $7.1 \%(\mathrm{n}=2)$ had chronic urinary retention, 7.1\% $(\mathrm{n}=2)$ had spina bifida, 7.1\% $(\mathrm{n}=2)$ had dysfunctional voiding, 3.6\% $(\mathrm{n}=1)$ had Fowler syndrome, and 3.6\% $(\mathrm{n}=1)$ had chronic interstitial cystitis.

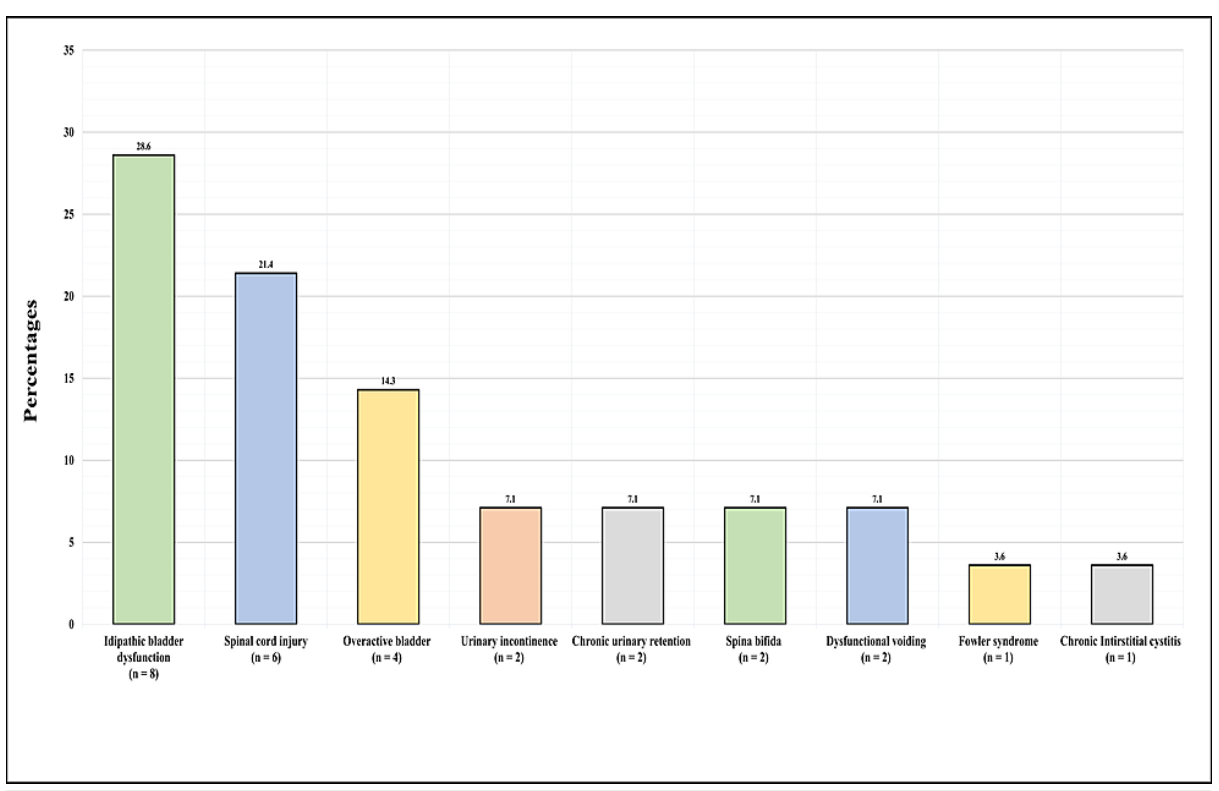

FIGURE 2: Primary diagnoses among participants.

Table 2 demonstrates the trials done with the patients and the outcome. 46.4\% (n=13) Failed to progress from the evaluation phase to the implantation phase. 


\section{Cureus}

\begin{tabular}{|c|c|c|}
\hline & $\mathbf{n}$ & $\%$ \\
\hline \multicolumn{3}{|c|}{ Evaluation phase } \\
\hline Successful & 15 & 53.6 \\
\hline Unsuccessful & 13 & 46.4 \\
\hline \multicolumn{3}{|c|}{ Implantation phase } \\
\hline Not done & 16 & 57.1 \\
\hline Successful & 12 & 42.9 \\
\hline
\end{tabular}

\section{TABLE 2: Outcome of SNM trials and implantations.}

SNM: sacral neuromodulation.

Table 3 displays the factors associated with failure in the evaluation stage. No significant association was found between failure in the evaluation stage and gender, age, having a co-morbidity, and SNM indication.

\begin{tabular}{|c|c|c|c|}
\hline \multirow{2}{*}{ Predictor } & \multicolumn{2}{|c|}{ Evaluation trial outcome } & \multirow{2}{*}{ p-value } \\
\hline & Success & Failure & \\
\hline \multicolumn{4}{|l|}{ Gender } \\
\hline Male & $6(46.2 \%)$ & $7(53.8 \%)$ & \multirow[t]{2}{*}{0.464} \\
\hline Female & $9(60 \%)$ & $6(40 \%)$ & \\
\hline Age & $31.93+11.81$ & $43.15+15.67$ & 0.065 \\
\hline \multicolumn{4}{|l|}{ Presence of a comorbidity } \\
\hline No & $9(56.3 \%)$ & $7(43.8 \%)$ & \multirow[t]{2}{*}{0.743} \\
\hline Yes & $6(50 \%)$ & $6(50 \%)$ & \\
\hline \multicolumn{4}{|l|}{ Indication } \\
\hline Spinal cord condition & $2(25 \%)$ & $6(75 \%)$ & \multirow[t]{2}{*}{0.055} \\
\hline Bladder condition & $13(65 \%)$ & $7(35 \%)$ & \\
\hline${ }^{\star}$ Significant at level 0.05 & & & \\
\hline
\end{tabular}

TABLE 3: Factors associated with failure at evaluation stage.

\section{Discussion}

There is emerging evidence that other parameters, such as age, gender, SNM indication, and body mass index (BMI) could have a role in increasing the likelihood of failure to progress to the implantation stage. For instance, unlike our study which found no link between SNM indication and trial outcomes, a Turkish study evaluating the progression rates from the evaluation phase to the implantation phase stated that all the patients who failed to progress had an underlying diagnosis of an overactive bladder [3]. In the United States, a similar report has emerged stating a link between BMI and evaluation stage failure. The study also identified a higher chance of evaluation phase success among females compared to males which also contradicts our negative findings. Moreover, patients with an urgency incontinence had higher chances of evaluation phase success compared to patients with urgency-frequency [4].

Nowadays, sacral neuromodulation is widely used for the treatment of many diseases which include but are not limited to overactive bladder, urinary retention, and intractable constipation $[5,6]$. The SNM implantation usually occurs in two stages with testing for the symptoms between them [7]. In our study, we found that $53,6 \%$ of our patients had success in the evaluation stage and went on to the implantation stage. Compared to our results, other studies showed marginally higher success rates ranging between $76 \%$ and 
In regard to the predictors of success of the evaluation stage, we found that there is no sufficient link between SNM evaluation success and age, gender, comorbidities, or indication which is similar to a Dutch single-center experience which concluded that neither gender, patient age, history nor diagnosis were predictors of evaluation stage success [15]. Conversely, many other studies found that women were more likely to experience success at the evaluation stage and proceed to the implant stage $[4,16,17]$. Also, other studies found an association between age and success of SNM evaluation with younger patients having a better response to SNM than older patients [18,19]. Furthermore, some studies found that patients who had urge urinary incontinence as the indication for sacral neuromodulation showed a significantly higher success rate [20].

The presented study had some limitations. First, the retrospective study design is one of our limitations. We could only look at data that had been previously entered into the patients' medical records. Consequently, we had to rely on others for accurate and safe record keeping. Second, the population size of the study is smaller than other larger multicenter studies done globally which may not be representative of the whole SNM population in Saudi Arabia. Hence why further prospective, randomized, multicenter studies are needed to assess the failure rate in the evaluation stage of sacral neuromodulation and the predictors of success since there is a discrepancy in the studies that we found in the literature.

\section{Conclusions}

The observed failure rate was marginally higher than the ones detected globally. In contrast to previous studies, no sufficient link has been observed between failure in the evaluation stage and gender, age, having a co-morbidity, or SNM indication. Sacral neuromodulation is shown to be an advanced treatment of some chronic urinary system diseases. However, further studies are needed to establish a reliable result to improve the use of SNM and further refine its indication criteria in order to achieve the optimal results, especially in the region, i.e., Saudi Arabia. The magnitude of the effect comorbidities have on SNM can be better explained with the use of a performance status criteria, such as ECOG and NYHA FC, which the presented study failed to do so due to its small population size. Additionally, factors that could affect the subjective perception to the quality of therapy, such as the concomitant depression and anxiety in patients with bladder disorders need to be further explored as they might affect the patients' satisfaction during the evaluation phase, thereby improving patients' quality of life.

\section{Additional Information \\ Disclosures}

Human subjects: Consent was obtained or waived by all participants in this study. King Abdullah International Medical Research Center issued approval NRC21R/095/03. The Institutional Review Board of King Abdullah International Medical Research Center, the Ministry of National Guard-Health Affairs, Riyadh, Kingdom of Saudi Arabia, approved the study with approval number NRC21R/095/03. The patients' confidentiality and anonymity was ensured, as serial numbers replaced the medical record numbers. The data was accessed and used by only the research team. . Animal subjects: All authors have confirmed that this study did not involve animal subjects or tissue. Conflicts of interest: In compliance with the ICMJE uniform disclosure form, all authors declare the following: Payment/services info: All authors have declared that no financial support was received from any organization for the submitted work. Financial relationships: All authors have declared that they have no financial relationships at present or within the previous three years with any organizations that might have an interest in the submitted work. Other relationships: All authors have declared that there are no other relationships or activities that could appear to have influenced the submitted work.

\section{References}

1. Leong RK, De Wachter SG, van Kerrebroeck PE: Current information on sacral neuromodulation and botulinum toxin treatment for refractory idiopathic overactive bladder syndrome: a review. Urol Int. 2010, 84:245-53. 10.1159/000288223

2. Sukhu T, Kennelly MJ, Kurpad R: Sacral neuromodulation in overactive bladder: a review and current perspectives. Res Rep Urol. 2016, 8:193-9. 10.2147/RRU.S89544

3. Feldkamp A, Amasyalı AS, Groegler J, Jellison F, Staack A: Evaluation of progression from first to second stage sacral neuromodulation and unplanned device removal. Turk J Urol. 2021, 47:58-65. 10.5152/tud.2020.20288

4. Faris AE, Gill BC, Pizarro-Berdichevsky J, et al.: Impact of age and comorbidities on use of sacral neuromodulation. J Urol. 2017, 198:161-6. 10.1016/j.juro.2017.02.020

5. Bernstein AJ, Peters KM: Expanding indications for neuromodulation. Urol Clin North Am. 2005, 32:59-63. 10.1016/j.ucl.2004.10.002

6. Zheng J, Li S, Nie Y, et al.: Sacral neuromodulation in the treatment of intractable constipation . Zhonghua Wei Chang Wai Ke Za Zhi. 2014, 17:1175-8. 10.3760/cma.j.issn.1671-0274.2014.12.005

7. Das AK, White MD, Longhurst PA: Sacral nerve stimulation for the management of voiding dysfunction . Rev Urol. 2000, 2:43-60.

8. Anger JT, Cameron AP, Madison R, Saigal C, Clemens JQ: Outcomes of sacral neuromodulation in a privately 
insured population. Neuromodulation. 2016, 19:780-4. 10.1111/ner.12472

9. Ismail S, Chartier-Kastler E, Perrouin-Verbe MA, Rose-Dite-Modestine J, Denys P, Phé V: Long-term functional outcomes of S3 sacral neuromodulation for the treatment of idiopathic overactive bladder. Neuromodulation. 2017, 20:825-9. 10.1111/ner.12696

10. Yazdany T, Bhatia N, Nguyen J: Determining outcomes, adverse events, and predictors of success after sacral neuromodulation for lower urinary disorders in women. Int Urogynecol J. 2011, 22:1549-54. 10.1007/s00192-011-1512-2

11. Gandhi S, Gajewski JB, Koziarz A, Almutairi S, Ali A, Cox A: Long-term outcomes of sacral neuromodulation for lower urinary tract dysfunction: A 23-year experience. Neurourol Urodyn. 2021, 40:461-9. 10.1002/nau.24583

12. Kaaki B, Gupta D: Medium-term outcomes of sacral neuromodulation in patients with refractory overactive bladder: A retrospective single-institution study. PLoS One. 2020, 15:e0235961.

10.1371/journal.pone.0235961

13. Morgan TN, Pace N, Mohapatra A, et al.: Sacral neuromodulation: determining predictors of success . Urology. 2021, 153:124-8. 10.1016/j.urology.2020.06.023

14. Pizarro-Berdichevsky J, Gill BC, Clifton M, Okafor HT, Faris AE, Vasavada SP, Goldman HB: Motor response matters: optimizing lead placement improves sacral neuromodulation outcomes. J Urol. 2018, 199:1032-6. 10.1016/j.juro.2017.11.066

15. Koldewijn EL, Rosier PF, Meuleman EJ, Koster AM, Debruyne FM, van Kerrebroeck PE: Predictors of success with neuromodulation in lower urinary tract dysfunction: results of trial stimulation in 100 patients. J Urol. 1994, 152:2071-5. 10.1016/s0022-534732311-x

16. Jairam R, Drossaerts J, Marcelissen T, van Koeveringe G, Vrijens D, van Kerrebroeck P: Predictive factors in sacral neuromodulation: a systematic review. Urol Int. 2021, 1-21. 10.1159/000513937

17. Nguyen LN, Bartley J, Killinger KA, et al.: Does sex matter? A matched pairs analysis of neuromodulation outcomes in women and men. Int Urol Nephrol. 2018, 50:825-32. 10.1007/s11255-018-1843-8

18. Sherman ND, Jamison MG, Webster GD, Amundsen CL: Sacral neuromodulation for the treatment of refractory urinary urge incontinence after stress incontinence surgery. Am J Obstet Gynecol. 2005, 193:2083-7. 10.1016/j.ajog.2005.07.038

19. Amundsen CL, Romero AA, Jamison MG, Webster GD: Sacral neuromodulation for intractable urge incontinence: are there factors associated with cure?. Urology. 2005, 66:746-50. 10.1016/j.urology.2005.04.031

20. Arlen AM, Powell CR, Kreder KJ: Sacral neuromodulation for refractory urge incontinence is less effective following spinal surgery. ScientificWorldJournal. 2011, 11:142-6. 10.1100/tsw.2011.13 